\title{
The Role of Radiative Surface Modes and Longitudinal Excitons in the Formation of Exciton-Polariton Luminescence Spectra of CdS-Type Crystals
}

\author{
Bozorboy J. Akhmadaliev, Nosirjon Kh. Yuldashev, Iftikhorjon I. Yulchiev \\ Ferghana Polytechnic Institute, Ferghana, Uzbekistan \\ Email: uzferfizika@mail.ru
}

How to cite this paper: Akhmadaliev, B.J., Yuldashev, N.Kh. and Yulchiev, I.I. (2018) The Role of Radiative Surface Modes and Longitudinal Excitons in the Formation of Exciton-Polariton Luminescence Spectra of CdS-Type Crystals. Optics and Photonics Journal, 8, 50-65.

https://doi.org/10.4236/opj.2018.83006

Received: February 20, 2018

Accepted: March 27, 2018

Published: March 30, 2018

Copyright $\odot 2018$ by authors and Scientific Research Publishing Inc. This work is licensed under the Creative Commons Attribution International License (CC BY 4.0).

http://creativecommons.org/licenses/by/4.0/

\begin{abstract}
The low-temperature ( $T=2 \mathrm{~K}$ ) exciton-polariton luminescence (EPL) spectra in the vicinity of the exciton-resonance frequency $A_{n=1}$ for CdS-type crystals have been theoretically and experimentally investigated with allowance for the mechanical exciton decay $\hbar \Gamma$. The results of the numerical calculations of the partial and interference contributions of the bulk and radiative surface spectral modes to the EPL in the geometry of additional s- and p-polarized waves emitted into vacuum are analyzed. It is shown that the contributions of purely longitudinal excitons and their interference with polaritons of the upper dispersion branch near the longitudinal frequency $\omega_{L}$ to the EPL are small $(\sim 10 \%-30 \%)$; nevertheless, they must be taken into account to obtain quantitative agreement with experimental data. Specifically these contributions are responsible for the formation of an additional $A_{L}^{\prime}$ line (along with the fundamental $A_{T}$ line) in the case of oblique incidence of radiation.
\end{abstract}

\section{Keywords}

Exciton-Polariton Luminescence, Spatial Dispersion, CdS, Exciton Decay, Longitudinal Excitons, Radiative Surface Spectral Modes, Exciton Green's Functions, Keldysh Diagram Technique, The Partial Contributions and Interference Component of the Luminescence

\section{Introduction}

The polariton mechanism of luminescence plays an important role in the formation of low-temperature emission spectra of semiconductor crystals in the vicin- 
ity of exciton resonances. To date, many fundamental questions concerning the polariton luminescence (PL), which are related to the specific features of polariton dispersion, conditions of radiation transmission through a boundary in the vicinity of resonance, and the character of spatial and energetic distribution of polaritons, have been understood [1]-[9]. A detailed study of the exciton energy structure of crystals shows that, along with the phenomena of exciton-polariton interaction (polariton effect) and spatial dispersion (dependence of the permittivity tensor $\varepsilon_{\alpha \beta}$ on the wave vector $\boldsymbol{k}$ ), the effects related to exciton decay may play an important role in the formation of low-temperature photoluminescence spectra. In particular, exciton decay may cause build-up of the emission of radiative surface modes (purely spatially decaying waves, propagating into crystal bulk at $\Gamma=0)$ and the interference luminescence of coherently emitting states of different dispersion branches with close $\omega$ and $\boldsymbol{k}$ values. A PL theory was developed in [6] [9] for the case where dissipative exciton decay may violate the applicability criteria for the kinetic Boltzmann equation

$$
\left|\operatorname{Re} \boldsymbol{k}_{\beta}\right| \gg \alpha_{\beta}
$$

This theory proved to be valid at an arbitrary relation between $\left|\operatorname{Re} \boldsymbol{k}_{2}\right|$ and $\alpha_{2}$ (where $\boldsymbol{k}_{2}$ and $\alpha_{2}$ are, respectively, the wave vector and the absorption coefficient for polaritons of upper dispersion branch 2). The contributions of the waves of lower polariton branch 1 and strongly damped wave 2 to the PL in the vicinity of longitudinal exciton frequency $\omega_{L}$ (branch 3 ) were successively calculated and the experimental spectra of $\mathrm{ZnP}_{2}$, CdS, and CdTe crystals were adequately analyzed based on this theory. However, the specific calculation and experiment were performed for the simplest case of normal polariton emission from a crystal into vacuum, where the contribution of longitudinal excitons to PL is zero. At the same time, it has been known for a long time (see, e.g., [3] [4] [5]) that energy transfer can be implemented in crystals by purely longitudinal excitons in the presence of spatial dispersion (SD). These excitons manifest themselves in low-temperature photoluminescence spectra of CdS crystals only in the case of oblique emission [7] [8]. Nevertheless, their contributions to the EPL have not been quantitatively analyzed.

In this study, the microscopic PL theory for CdS-type crystals with SD is generalized with allowance for the contributions of the emission of purely longitudinal excitons in the vicinity of frequency $\omega_{L}$. The EPL is considered for the case where inequality (1) is violated simultaneously for the additional waves $\beta=2$ and $\beta=3$ but remains valid for the polaritons of the lower dispersion branch $\beta=1$. The developed EPL theory, in contrast to the conventional PL theory based on the use of polariton distribution function, allows one to take into account the interference of coherently emitting states of transverse polaritons and longitudinal excitons in the presence of decay $\Gamma \neq 0$ (this is undoubtedly a new effect, which was not considered in [6] [7] [9] [10] [11]), i.e., go beyond the applicability limits of the kinetic equation for waves 2 and 3, which is also determined, along with (1), by a more stringent condition: 


$$
\left|\operatorname{Re}\left(\boldsymbol{k}_{\beta}-\boldsymbol{k}_{\beta}^{\prime}\right)\right| \gg \alpha_{\beta}, \alpha_{\beta^{\prime}}\left(\beta, \beta^{\prime}=2,3\right)
$$

If inequality (2) is valid, one can neglect the interference contribution of coherently emitting polariton states of different dispersion branches. Below, using this theory, we analyzed the angular dependence of experimental EPL spectra of CdS crystals with hexagonal point symmetry for $s$ - and $p$-polarized radiation.

\section{Theory}

\subsection{Statement of the Problem}

Let us consider a dipole-active triplet exciton, which is characterized by the resonance frequency $\omega_{0}$, effective mass $M$, and longitudinal-transverse splitting $\omega_{L T}=\omega_{L}-\omega_{0}$, in a crystal with isotropic permittivity tensor [5] [6] [9]

$$
\varepsilon_{\alpha \beta}(\omega, \boldsymbol{k})=\varepsilon(\omega, k) \delta_{\alpha \beta}=\varepsilon_{b}\left[1+\frac{\omega_{L T}}{\omega_{o}(k)-\omega-i \Gamma(\omega, k) / 2}\right] \cdot \delta_{\alpha \beta}
$$

where $\varepsilon_{b}$ is the background permittivity; $\hbar \omega_{o}(k)=\hbar \omega_{o}+\frac{\hbar^{2} k^{2}}{2 M}$ is the mechanical exciton energy; and $\Gamma(\omega, k)$ is the exciton decay, which is determined by scattering or capture processes. It is known that normal waves of two types can be excited in these crystals: transverse $\left(\varepsilon(\omega, k)=c^{2} k^{2} / \omega^{2}\right.$, where $c$ is the speed of light in vacuum) and longitudinal $(\varepsilon(\omega, k)=0)$. For most semiconductors $\omega_{L T} \ll \omega_{0}$ (for example, $\omega_{L T}=2.0 \mathrm{meV}$ and $\omega_{0}=2552.4 \mathrm{meV}$ for CdS) and in the vicinity of the resonance $\omega_{0}$, which is determined by the condition

$$
\left|\omega-\omega_{o}\right| \ll \sqrt{\omega_{o} \omega_{L T}}
$$

the dispersion equations of transverse polaritons $(\beta=1,2)$ and longitudinal excitons $(\beta=3)$ can be presented in a simplified form:

$$
\begin{aligned}
& \omega=\omega_{\mathbf{k}}^{(T)}=\omega_{o}+\frac{\hbar k^{2}}{2 M}-\frac{\varepsilon_{b} \omega_{L T}}{\left(c k / \omega_{o}\right)^{2}-\varepsilon_{b}}-i \frac{\Gamma(\omega, k)}{2} \\
& \omega=\omega_{\mathbf{k}}^{(3)}=\omega_{L}+\frac{\hbar k^{2}}{2 M}-i \frac{\Gamma(\omega, k)}{2}, \quad \omega_{L}=\omega_{o}+\omega_{L T}
\end{aligned}
$$

Note that polaritons 1 and 2 are described by formula (5) in the ranges $k>k_{0} \sqrt{\varepsilon_{b}}$ and $k<k_{0} \sqrt{\varepsilon_{b}}$, respectively $\left(k_{0}=\omega_{0} / c\right)$.

Figure 1 shows calculated exciton-polariton dispersion curves in the case of isolated exciton resonance with isotropic effective mass for the following $\hbar \Gamma$ values: 1) 0.075 , 2) 0.5 , 3) 1.0 , 4) 1.457 , 5) 2.0 , and 6) $5.0 \mathrm{meV}$. As can be seen in the figure, the dispersion curves of exciton-polariton modes are significantly transformed with an increase in decay $\hbar \Gamma$. This transformation is especially pronounced near the longitudinal frequency $\omega_{L}$ for modes $2,2^{\prime}$ and 3,3 , which can propagate only in the frequency range of $\omega>\omega_{\theta}$ and are purely decaying (i.e., radiative surface) when $\omega<\omega_{\theta}$. The characteristic frequency $\omega_{\theta} \geq \omega_{L}$ is determined by the condition of total internal reflection for waves $\beta=2,3$ 

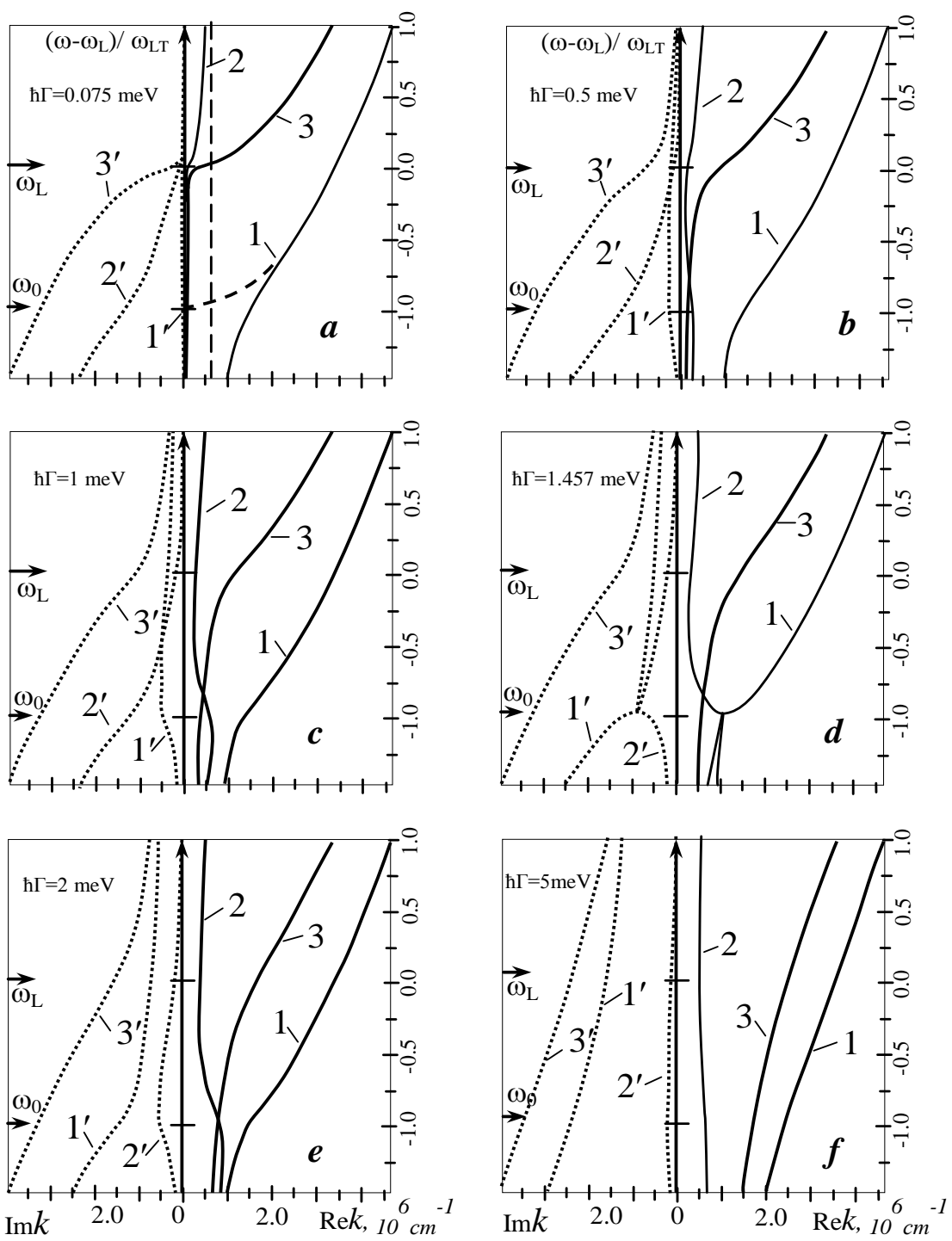

Figure 1. Calculated energy spectrum of normal waves depending on the exciton decay $\hbar \Gamma$ in a cubic crystal with SD for the following parameters of the lowest exciton resonance A $(n=1)$ in CdS: $\hbar \omega_{0}=2552.4 \mathrm{meV}, \hbar \omega_{L T}=2.0 \mathrm{meV}, M=0.9 m_{0}\left(m_{0}\right.$ is the free-electron mass), and $\varepsilon_{b}=9.4$ [7]. The dashed lines correspond to noninteracting mechanical excitons and photons: 1, 1', 2, 2' are transverse polaritons and 3, 3' are longitudinal excitons.

$$
\operatorname{Re} n_{\beta}=c \operatorname{Re} k_{\beta}(\omega) / \omega=\sin \theta
$$

Here, $\theta$ is the exit angle of radiation from the crystal into vacuum and $n_{\beta}$ is the refractive index of the crystal for wave $\beta\left(\operatorname{Re} n_{\beta}<1\right.$ at $\left.\omega<\omega_{\theta}\right)$. We should note the following. First, the occurrence of an imaginary part of wave vector $k_{\beta}$ at $\hbar \Gamma \neq 0$ (Figure 1 , curves $2^{\prime}, 3^{\prime}$ ) at $\omega>\omega_{\theta}$ indicates a certain spatial decay of propagating modes, which leads (in some frequency range $\omega-\omega_{\theta} \sim \Gamma$ ) to violation of inequalities (1) and (2). Second, in the frequency range $\omega<\omega_{\theta}$, where condition (1) is always violated for waves 2 and 3 , a real additive to wave vector $k_{\beta}$ arises at finite $\Gamma$ (curves 2 and 3 ); this additive indicates that radiative 
surface modes 2 and 3 cease to be purely decaying and are involved in the transfer of exciton excitation energy in the crystal. The latter circumstances are undoubtedly of great importance for the formation of the EPL spectrum of crystals in the emission geometry of purely longitudinal excitons, whose analysis is the object of our study.

Let us analyze the spectral intensity $I_{\lambda}^{(0)}(\omega, \Omega)$ of radiation with polarization $\lambda$, propagating in vacuum near the crystal surface along the unit vector $\Omega(\theta, \varphi)$ (which lies in the $y z$ plane, while the internal normal to the crystal boundary is directed along the $z$ axis; $\lambda=s, p$, where $s / / x, p / / y)$ :

$$
I_{\lambda}^{(0)}(\omega, \boldsymbol{\Omega})=\sum_{\beta} I_{\beta \lambda}^{(0)}(\omega, \boldsymbol{\Omega}) .
$$

Here, $\beta=1,2$ at $\lambda=s$ and $\beta=1,2,3,23$ at $\lambda=p$. The partial intensity $I_{23 p}^{(0)}$ describes the interference contribution of waves 2 and 3 to the EPL. We assume that low-temperature luminescence is excited via stationary illumination of crystal by natural light in the intrinsic absorption spectral region ( $\hbar \omega \geq E_{g}$ ), where free electrons and holes are generated. Being thermalized, these charge carriers are coupled (as a result of the Coulomb interaction) into excitons, which, in turn, are multiply scattered from phonons, impurities, and other lattice defects to relax (in both energy and momentum) into the vicinity of resonance $\omega_{o}$, where, in terms of the conventional PL theory, some nonequilibrium stationary polariton distribution function is settled. Near the frequency $\omega_{L}$, the densities of states

$$
\rho_{\beta}(\omega)=\frac{1}{V_{o}} \sum_{\boldsymbol{k}} \delta\left(\omega-\omega_{\boldsymbol{k}}^{(\beta)}\right)=\frac{k_{\beta}^{2}(\omega)}{(2 \pi)^{3} v_{\beta}(\omega)},
$$

where $V_{o}$ is the crystal volume and $v_{\beta}(\omega)=\mathrm{d} \omega / \mathrm{d} k_{\beta}$ is the polariton group velocity, are significantly different for the polaritons belonging to branch 1 and branches $\beta=2,3$. For example, at $\omega>\omega_{L}, \rho_{1}(\omega) / \rho_{2}(\omega) \approx\left(\omega_{L T} / \varepsilon_{b} \omega_{M}\right)^{3 / 2} \approx 3 \times 10^{3}$ for CdS $\left(\omega_{M}=\hbar k_{0}^{2} / 2 M\right)$. Then, naturally, the wave scattering within the branches $\beta=2,3$ can be disregarded in the vicinity of frequency $\omega_{L}$. For the same reason, one can neglect the generation of waves 2 and 3 with small $\boldsymbol{k}$ values (Figure 1(a)), which occurs due to the scattering by $L O$ phonons from higher lying exciton states and due to their possible induction by multiple specular reflection of waves 1 from the internal crystal boundary [5]. Thus, the distribution function $f$ of polaritons is formed mainly due to their relaxation over the states of the transverse mode $1-f_{1 k \lambda}(\omega, z)$.

Since the successive determination of the function $f_{1 k \lambda}(\omega, z)$ is beyond the scope of our study, this function is considered as specified. Naturally, isotropization of the distribution function over directions and polarization states occurs in a cubic crystal as a result of multiple scattering. Furthermore, when carrying out a calculation in a narrow frequency range near $\omega_{L}$, it is sufficient to restrict oneself to the simplest factorized form

$$
f_{1 k \lambda}(\omega, z)=f_{1}(\omega) \exp (-z / L)
$$


which contains some smooth frequency dependence $f_{1}(\omega)$. Here, $L$ is the effective distribution depth for the polaritons of branch 1 , which is determined by the processes of multiple scattering of free carriers and excitons-polaritons. The coordinate dependence $f_{1 k \lambda}(\omega, z)$ on only $z$ characterizes the problem as homogeneous along the surface plane.

The partial contribution $I_{1 \lambda}^{(0)}$ is determined from the formula

$$
I_{1 \lambda}^{(0)}(\omega, \Omega)=\frac{k_{o}^{2}}{(2 \pi)^{3}} \hbar \omega T_{01}^{(\lambda)} \cdot f_{1 k \lambda}(z=+0)
$$

where

$$
T_{o \beta}^{(\lambda)}=\frac{I_{N}^{(0)}}{I_{N}^{(\beta)}}=\frac{\cos \theta}{\cos \theta_{\beta}} \frac{1}{n_{\beta}} \frac{w_{0}}{w_{\beta}}\left|t_{o \beta}^{(\lambda)}\right|
$$

is the energy transmittance of wave $\beta$ with polarization $\lambda$, emerging from the crystal into vacuum, and $t_{o \beta}^{(\lambda)}$ is the corresponding amplitude transmittance. In expression (8), $I_{N}$ is the normal component of the energy fluence to the surface and $n_{\beta} w_{\beta}$ is the coupling coefficient between the energy fluence and the squared modulus of the electric field amplitude for the normal wave $\beta$ :

$$
w_{o}=\frac{c}{8 \pi}, \quad w_{\beta}=\frac{c}{8 \pi}\left[1-\delta_{\beta 3}+\frac{\omega_{M} \varepsilon_{b}}{\omega_{L T}}\left(\frac{n_{\beta}^{2}}{\varepsilon_{b}}-1+\delta_{\beta 3}\right)^{2}\right], \quad \beta=1,2,3 .
$$

\subsection{Theoretical Calculation}

The intensity of $s$-polarized EPL,

$$
I_{s}^{(o)}(\omega, \boldsymbol{\Omega})=I_{1 s}^{(o)}(\omega, \boldsymbol{\Omega})+I_{2 s}^{(o)}(\omega, \boldsymbol{\Omega})
$$

does not contain the interference term, because condition (2) is assumed to be satisfied. The partial contribution $I_{1 s}^{(o)}$ is determined in the kinetic approximation from formula (8), where $\lambda=s$. To calculate $I_{2 s}^{(o)}$ at finite exciton decays, i.e., with allowance for the arbitrary relation between $\operatorname{Re} k_{2}$ and $\alpha_{2}$, we will use the Keldysh diagram technique and find the Green's function $G_{2 S}^{-+}\left(\boldsymbol{k}, \boldsymbol{k}^{\prime} ; \omega\right)$ for polaritons of branch 2 with polarization $\lambda$ at a specified distribution function for polaritons of branch 1 , neglecting the scattering in branch 2 . Figure 2 shows the corresponding diagram for the function $G_{\beta \lambda}^{-+}\left(\boldsymbol{k}, \boldsymbol{k}^{\prime} ; \omega\right)$ (here, $\beta=2$ ). The solid lines present the exciton Green's functions, renormalized with allowance for the exciton-photon and exciton-lattice interactions

$$
\begin{gathered}
G_{\boldsymbol{k} \omega}^{--}=\left[\omega-\omega_{\mathbf{k}}^{(T)}+i \Gamma(\omega, \boldsymbol{k}) / 2\right]^{-1}, \quad G_{\boldsymbol{k} \omega}^{++}=-\left(G_{\boldsymbol{k} \omega}^{--}\right)^{*}, \\
G_{1 \boldsymbol{k} \lambda \omega}^{-+}(z)=2 \pi f_{1 \boldsymbol{k} \lambda}(z) \delta\left(\omega-\omega_{1 \mathbf{k}}^{(T)}\right),
\end{gathered}
$$

where $\Gamma(\omega, \boldsymbol{k})$ is the polariton decay, determined by the scattering or capture processes. The function $G_{\mathbf{k} \omega}^{--}\left(G_{\boldsymbol{k} \omega}^{++}\right)$is expanded in a sum of two pole terms:

$$
G_{\boldsymbol{k} \omega}^{--}=\sum_{\beta=1,2} G_{\beta \boldsymbol{k} \omega}^{--}, \quad G_{\beta \boldsymbol{k} \omega}^{--}=(-1)^{\beta} \frac{2 M}{\hbar} \frac{k_{\beta}^{2}-\varepsilon_{b} k_{0}^{2}}{k_{1}^{2}-k_{2}^{2}} \frac{1}{k^{2}-k_{\beta}^{2}} .
$$




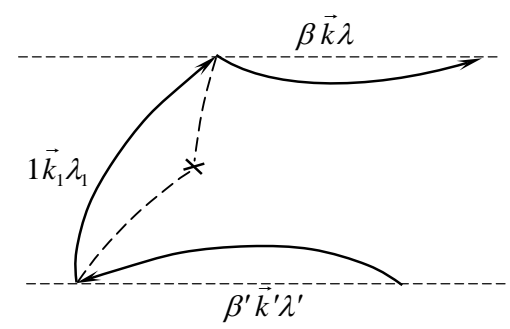

Figure 2. Diagram for the Green's function $G_{\beta \lambda}^{-+}\left(\boldsymbol{k}, \boldsymbol{k}^{\prime} ; \omega\right): \beta=\beta^{\prime}=2$ at $\lambda=s$ and $\beta, \beta^{\prime}=2,3$ at $\lambda=p$.

When calculating $I_{2 s}^{(o)}$, the functions $G_{1 \mathbf{k} \omega}^{--}$and $G_{1 \mathbf{k}^{\prime} \omega}^{++}$are assigned to the upper and lower exciton lines in Figure 2. It is not necessary to take into account the diagram with external lines $G_{1 k \omega}^{--}$and $G_{1 k \omega}^{++}$(i.e., to consider the $1 \rightarrow 1$ scattering), because the function $f_{1 k \lambda}(z)$ is assumed to be specified. Using the diagram technique rules, we obtain

$$
\begin{gathered}
I_{2 S}^{(0)}(\omega, \boldsymbol{\Omega})=\frac{1}{8 \pi} \cdot \frac{k_{0}^{2}}{(2 \pi)^{3}}\left|t_{02}^{(S)}\right|^{2}\left\langle\left|E_{S}^{(2)}\left(\boldsymbol{k}_{\perp}, \omega ; z=+0\right)\right|^{2}\right\rangle, \\
\left\langle\left|E_{S}^{(2)}\left(\boldsymbol{k}_{\perp}, \omega ; z\right)\right|^{2}\right\rangle \\
=C \sum_{\boldsymbol{k}_{1} \lambda_{1}} \sum_{j}\left|\sum_{k_{z}} \frac{G_{2 \boldsymbol{k} \omega}^{--}}{n_{2}^{2}(\omega)-\varepsilon_{b}} \exp \left[i k_{z}\left(z-z_{j}\right)\right] e_{S}^{\left(\boldsymbol{k}_{1} \lambda_{1}\right)} \cdot V\left(\boldsymbol{k}_{1}-\boldsymbol{k}_{2}\right)\right|^{2} G_{1 \boldsymbol{k}_{1} \lambda_{1} \omega}^{-+}(z)
\end{gathered}
$$

Here, $E_{S}^{(\beta)}\left(\boldsymbol{k}_{\perp}, \omega ; z\right)$ is the amplitude of a light wave with frequency $\omega$ and transverse component $\boldsymbol{k}_{\perp}$ (note that $\boldsymbol{k}_{\perp}$ is a good quantum number), equal to the transverse component $k_{o} \boldsymbol{\Omega}_{\perp}$ of the wave vector of this wave in vacuum; $t_{o \beta}^{(s)}$ is the amplitude transmittance; $n_{\beta}=k_{\beta} / k_{0}$ is the refractive index of wave $\beta ; e^{\left(k_{1} \lambda_{1}\right)}$ is the polarization vector; $e_{S}^{\left(k_{1} \lambda_{1}\right)}$ is its projection onto the $X$ axis; $Z_{j}$ is the coordinate of the $j$ th scattering center; and $C$ is a constant.

Substituting (9b) into (9a), replacing summation over $j$ with integration over $\boldsymbol{r}_{j}$ according to the rule $\sum_{j} \rightarrow N_{i} \int \mathrm{d} \boldsymbol{r}_{j}$, and integrating over $k_{z}$ and $\boldsymbol{k}_{1}$, we finally arrive at

$$
I_{2 S}^{(o)}(\omega, \boldsymbol{\Omega})=\frac{\left|t_{02}^{(s)}\right|^{2}}{(2 \pi)^{3}} \frac{f_{1}(\omega)}{2 k_{o} \operatorname{Im} n_{2 z}+L^{-1}} \frac{2 M^{2} c \varepsilon_{b} \omega_{L T} \cos \theta}{\hbar \tau_{21}\left|n_{2 z}\left(n_{1}^{2}-n_{2}^{2}\right)\right|^{2}},
$$

where $n_{2 z}=\left(n_{2}^{2}-\sin ^{2} \theta\right)^{1 / 2}$; the values of $t_{02}^{(s)}$ and $n_{2}$ are calculated with allowance for the decay $\Gamma=1 / 2 \tau_{21}$.

In the case of elastic scattering from static defects,

$$
\frac{1}{\tau_{21}(\omega)}=\frac{2 \pi}{\hbar^{2}} \frac{2}{3} N_{i} \sum_{\boldsymbol{k}_{1}}\left|V\left(\boldsymbol{k}_{1}-\boldsymbol{k}_{2}\right)\right|^{2} \cdot \delta\left(\omega-\omega_{1 k_{1}}^{(T)}\right),
$$

where $N_{i}$ is the defect concentration and $V(\boldsymbol{q})$ is the Fourier transform of the perturbing potential. For an isotropic potential, $V(\boldsymbol{q}) \equiv V(q)$. Note that, at $\omega_{M} \ll \omega_{L T} / \varepsilon_{b}$, the $k_{2}(\omega)$ value is small in comparison with $k_{1}(\omega)$, and the term 
$V\left(\boldsymbol{k}_{1}-\boldsymbol{k}_{2}\right)$ in (9c) can be replaced with $V\left(\boldsymbol{k}_{1}\right)$.

In the case of $p$-polarized radiation, to calculate the partial contributions $I_{2 p}^{(0)}, I_{3 p}^{(0)}$, and the interference component $I_{p 23}^{(0)}$ to the total intensity $I_{p}^{(0)}$, using formula (8),

$$
I_{p}^{(0)}(\omega, \theta)=\sum_{\beta=1,2,3} I_{\beta p}^{(0)}(\omega, \theta)+I_{p 23}^{(0)}(\omega, \theta)
$$

We will consider the Green's functions $G_{2 p}^{-+}\left(\boldsymbol{k}, \boldsymbol{k}^{\prime} ; \omega\right)$ (for the transverse polaritons of branch 2) and $G_{3 p}^{-+}\left(\boldsymbol{k}, \boldsymbol{k}^{\prime} ; \omega\right)$ (for longitudinal excitons). The diagram for these functions is shown in Figure 2, where $\lambda, \lambda^{\prime}=p$ and $\beta, \beta^{\prime}=2,3$. The solid line in the case of longitudinal exciton corresponds to the Green's function

$$
G_{3 k \omega}^{--}=\left[\omega-\omega_{k}^{(3)}+i \Gamma(\omega, \boldsymbol{k}) / 2\right]^{-1}=-\frac{2 M}{\hbar} \frac{1}{k^{2}-k_{3}^{2}},
$$

where $\omega_{k}^{(3)}$ is determined from formula (6), and $k_{3}$ is a solution to the dispersion equation for longitudinal excitons:

$$
k_{3}=n_{3} k_{0}, \quad n_{3}=\left(\left(\omega+i \frac{\Gamma}{2}-\omega_{L}\right) / \omega_{M}\right)^{1 / 2} .
$$

Using the corresponding considerations and calculation technique for the $s$ polarization, we arrive at

$$
\begin{gathered}
I_{\beta p}^{(0)}(\omega, \theta)=F_{\beta} \cdot \frac{\left|F_{\beta p}\right|^{2}}{2 k_{0} \operatorname{Im} n_{\beta z}+L^{-1}}, \quad \beta=2,3, \\
I_{p 23}^{(0)}(\omega, \theta)=2 \operatorname{Re}\left[F_{23} \cdot \frac{F_{2 p} \cdot F_{3 p}^{*}}{-i k_{0}\left(n_{2 z}-n_{3 z}^{*}\right)+L^{-1}}\right],
\end{gathered}
$$

where

$$
\begin{aligned}
& F_{\beta}=\frac{1}{(2 \pi)^{3}} \frac{2 M^{2} c \varepsilon_{b} \omega_{L T} \cos \theta f_{i}(\omega)}{\hbar \tau_{\beta 1}}, \\
& F_{2 p}=\frac{t_{02}^{(p)}}{n_{2 z}\left(n_{1}^{2}-n_{2}^{2}\right)}, \quad F_{3 p}=\frac{t_{03}^{(p)}}{n_{3 z} \varepsilon_{b}},
\end{aligned}
$$

and $F_{23}$ is obtained from $F_{\beta}$ by replacing $\tau_{\beta 1}$ with $\left(\tau_{21} \cdot \tau_{31}\right)^{1 / 2}$. It was taken into account that the electric field strength and the exciton part of crystal polarization for longitudinal excitons are related as follows: $4 \pi \boldsymbol{P}_{3}=-\varepsilon_{b} \boldsymbol{E}_{3}$. Expression (14) describes the interference contribution of the waves of branches 2 and 3 to the intensity of external p-polarized radiation; this contribution is caused by scattering from the same polariton center of branch 1 into coherently emitting states 2 and 3 under conditions where inequality (2) is also violated, along with Equation (1).

Now we have to calculate the amplitude transmittances $t_{0 \beta}^{(p)}$ of wave $\beta$ into vacuum. To this end, we used, along with the boundary Maxwell conditions, Pekar additional boundary conditions with a "dead layer." For the case where 
p-polarized wave 2 falls on the crystal surface from the bulk, we find

$$
t_{02}^{(p)}(\theta)=\frac{n_{1}^{2}-n_{2}^{2}}{n_{1}^{2}-\varepsilon_{b}} \frac{2 n_{2 z} / n_{2}}{\left(n_{o z}+\bar{n}_{p}\right) \cos \delta-i \sin \delta \cdot\left[\frac{\tilde{n}_{z}}{\varepsilon_{b}}\left(1+\bar{n}_{p 1}\right)+\frac{n_{o z} \varepsilon_{b}}{\tilde{n}_{z}} \cdot \bar{n}_{p 2}\right]},
$$

where

$$
\begin{gathered}
\bar{n}_{p}=n_{o z} \bar{n}_{p 1}+\bar{n}_{p 2}, \quad \bar{n}_{p 2}=\left(n_{2 z}+n_{1 z} \cdot \bar{n}_{p 1}\right) \cdot \varepsilon_{b}, \\
\bar{n}_{p 1}=\frac{n_{1}^{2} n_{2 z} n_{3 z}+n_{o x}^{2}}{n_{2}^{2} n_{1 z} n_{3 z}+n_{o x}^{2}} \cdot \frac{\varepsilon_{b}-n_{2}^{2}}{n_{1}^{2}-\varepsilon_{b}}, \quad n_{\beta x}=n_{o x}=\sin \theta .
\end{gathered}
$$

The transmittance $t_{01}^{(p)}$ is obtained from $t_{02}^{(p)}$ by the symmetric replacement $n_{1} \leftrightarrow n_{2}$. When a longitudinal wave falls on the crystal surface from the bulk, we determine the amplitude coefficient of conversion of longitudinal-exciton normal wave into external light as follows:

$$
=\frac{t_{03}^{(p)}(\theta)}{\sum_{\beta=1,2}(-1)^{\beta} \frac{n_{\beta}^{2}-\varepsilon_{\beta}}{n_{\beta}^{2}}\left(n_{o x}^{2}+n_{\beta z} n_{3 z}\right) \cdot\left[\left(n_{o z}+\frac{n_{3-\beta, z}}{\varepsilon_{b}}\right) \cos \delta-i \sin \delta\left(\frac{\tilde{n}_{z}}{\varepsilon_{b}}+\frac{n_{o z} n_{3-\beta, z}}{\tilde{n}_{z}}\right)\right]}
$$

\section{Discussion of the Results of Numerical Calculation and Comparison with Experimental Data}

The partial $\left(I_{\beta p}^{(0)}(\omega), I_{\beta s}^{(0)}\right)$ and integrated $\left(I_{s}^{(0)}(\omega), I_{p}^{(0)}\right)$ luminescence spectral intensities were numerically calculated using formulas (8 - 10) and (13 - 15) as basic ones. Auxiliary formulas of the (16), (17) type were applied to calculate the amplitude transmittances $t_{0 \beta \lambda}$ and refractive indices $n_{\beta}$. The only variable parameter of the theory is the effective distribution depth $L$ for lower branch polaritons. The $\hbar \Gamma$ value was assumed to be known from experiment. Numerical calculations were performed with the following values of the main parameters of exciton resonance $A_{n=1}$ for CdS crystal: $\hbar \omega_{0}=2.5524 \mathrm{eV}$, $\hbar \omega_{L T}=2.0 \mathrm{meV}, M_{e x}=0.9 m_{0} \quad\left(m_{0}\right.$ is the free-electron mass), background permittivity $\varepsilon_{b}=9.4$, and “dead layer" thickness $\ell=70 \AA$. These values of parameters are in good agreement with the experimental results on exciton reflection of light and exciton luminescence at $T=2 \mathrm{~K}$ [4] [7].

Figure 3 shows the theoretical spectral dependences $I_{p}^{(0)}(a), I_{2 p}^{(0)}(b), I_{3 p}^{(0)}(c)$, and $I_{p 23}^{(0)}(d)$, calculated with different values of the $\Gamma$ and $L$ parameters. The intensity and half-width of the curves $I_{2 p}^{(0)}(\omega)$ and, correspondingly, $I_{p}^{(0)}(\omega)$ turned out to depend strongly on $\Gamma \cdot L$. Similar parameters of the curves $I_{3 p}^{(0)}(\omega)$ and $I_{p 23}^{(0)}(\omega)$ at $L>1 \mu \mathrm{m}$ are barely sensitive to variations in $L$, which is due to the relatively large absorption coefficient for wave $3\left(\alpha_{3} \cdot L \gg 1\right)$.

Moreover, the half-width $\Delta_{3}$ of $I_{3 p}^{(0)}(\omega)$ is practically completely determined by the $\Gamma$ value. According to the numerical calculation, $\Delta_{3} \approx 2 \Gamma$. Note that the contribution of $I_{3 p}^{(0)}$ to $I_{p}^{(0)}$ sharply decreases with an increase in $\hbar \Gamma$; at the 


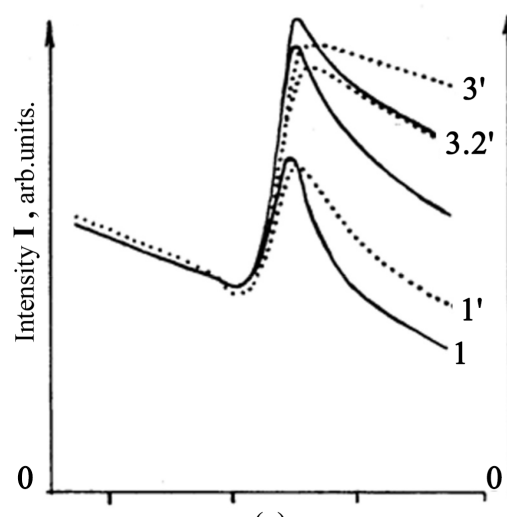

(a)

(c)

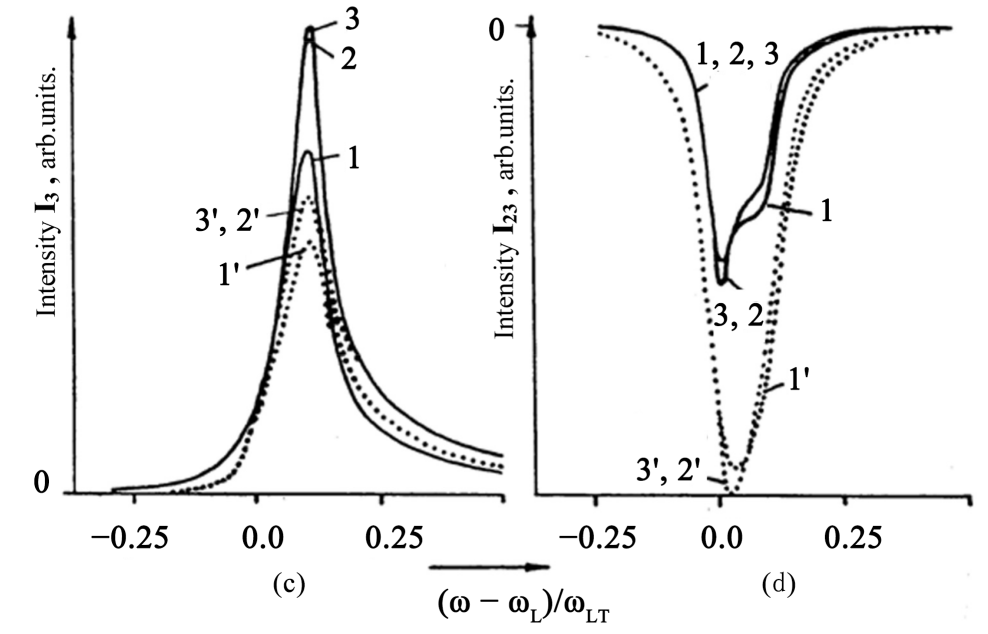

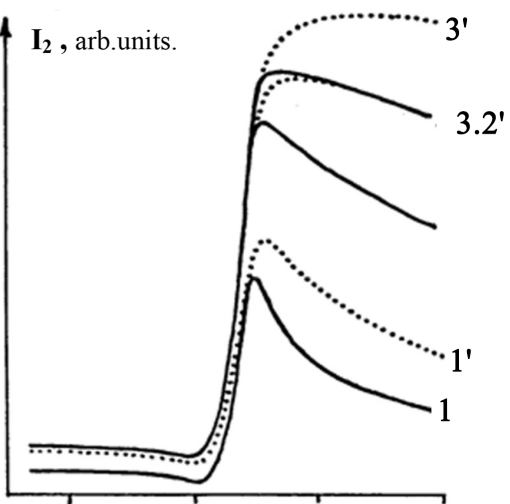

(b)

Figure 3. Spectral dependences of the total $(I)$ and partial $\left(I_{2 p}, I_{3 p}\right)$ intensities and the interference contribution $I_{p 23}$, calculated for the parameters of exciton resonance $A_{(n=1)}$ in CdS at the exit angle $\theta=80^{\circ}$ and different values of the exciton decay, $\hbar \Gamma=0.075(1-3)$ and $0.15\left(1^{\prime}-3^{\prime}\right) \mathrm{meV}$, and the distribution depth of branch-1 polaritons, $L=0.2\left(1,1^{\prime}\right), 1.0\left(2,2^{\prime}\right)$, and $2.0\left(3,3^{\prime}\right) \mu \mathrm{m}$.

same time, $I_{2 p}^{(0)}$ increases and significantly broadens. As a result, at the same $L$ value, a rise in $\Gamma$ leads to a slight decrease in the maximum of the dependence $I_{p}^{(0)}(\omega)$ at the frequency $\omega_{\theta}$ and its broadening. This is explained as a result of SD suppression for longitudinal waves by mechanical-exciton decay.

The quantum theory presented in Section 2 was used to describe the experi mental EPL spectra of CdS crystals in "isotropic" emission geometries ( $s$ polarization, when $\boldsymbol{\Omega}(\theta, \varphi)$ lies in the $x z$ plane and the optical axis $C / / X$, and $p$ polarization, when $\boldsymbol{\Omega} \perp \boldsymbol{C} / / Y$; here, the $z$ axis is perpendicular to the emitting crystal surface). Spectra were recorded on a setup developed by Professor A.V. Sel'kin (we kindly acknowledge his support). The setup was based on a DFS-24 spectrometer and operated in the photon-counting mode at a minimum spectral width of the slit $(0.04 \mathrm{meV})$, under conditions providing maximum spectral resolution. EPL excitation was performed at a wavelength $\lambda=476.5 \mathrm{~nm}$ by an $\mathrm{Ar}^{+}$ laser beam focused on the crystal surface into a spot $\sim 0.4 \times 4 \mathrm{~mm}^{2}$ in size at a luminous flux power of $7 \mathrm{~mW}$. 
Figure 4(a) and Figure 4(c) demonstrate the general view of the luminescence profiles for the CdS crystal in the $s$-emission geometry at (a) $\theta=0$ and (c) $\theta=80^{\circ}$. The dashed line in Figure $4(\mathrm{a})$ presents the experimentally measured (from the $2 \mathrm{LO}$ replica spectrum) occupancy function $g_{1}(\omega)=\rho_{1}(\omega) f_{1}(\omega)$ for the polariton states of branch 1; it was used to determine the distribution function $f_{1}(\omega)$, which is also shown in Figure 4 (a) by a dotted line. The theoretical EPL spectra in Figure 4(a') and Figure $4\left(\mathrm{c}^{\prime}\right)$ (curves 0 ), calculated at $\hbar \Gamma=0.075 \mathrm{meV}, L=0.8 \mu \mathrm{m}$, and $\delta=70 \AA$, are in good agreement with experiment (triangles). The partial contribution of $I_{2 S}^{(0)}$ (dotted lines 2) plays an

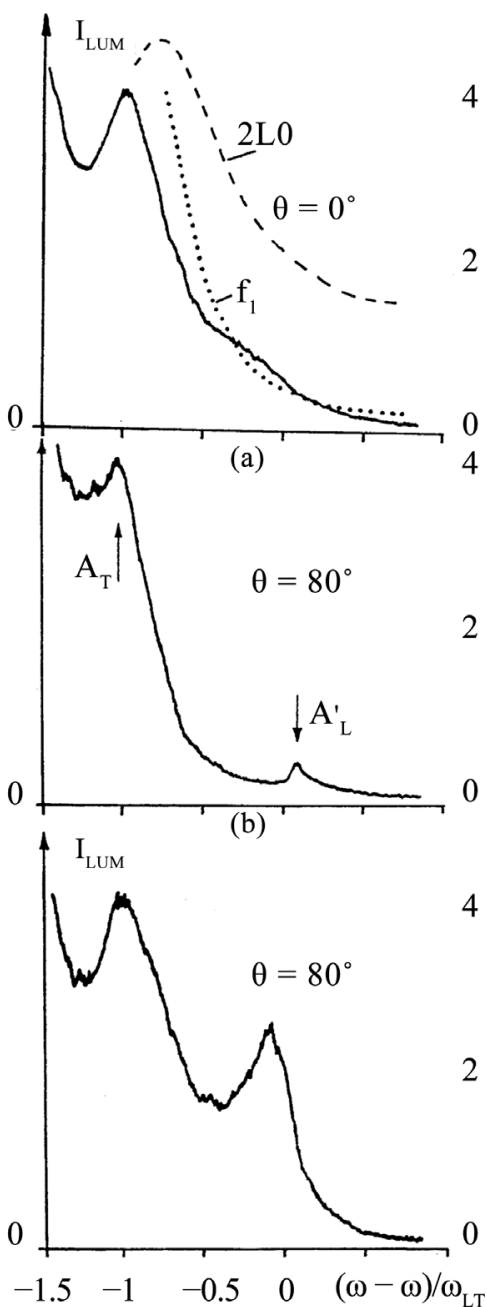

(c)

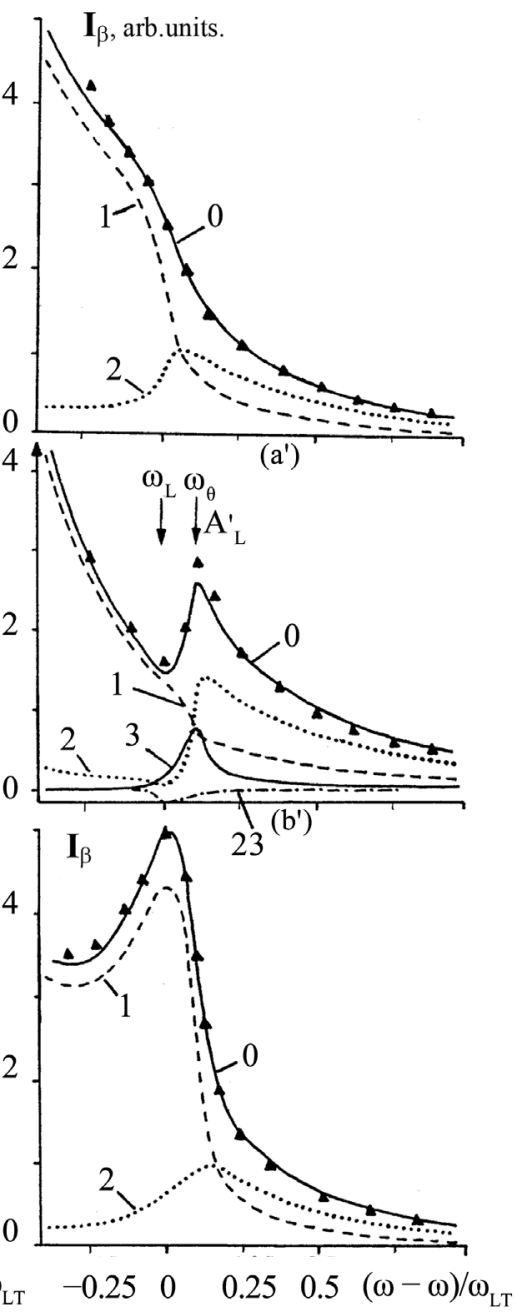

(c')

Figure 4. (a) (b) (c) Experimental and (a') (b'), (c') theoretical EPL spectra of CdS crystals $(\mathrm{T}=2 \mathrm{~K})$ for the detection geometries corresponding to the (a) (a') (c) (c') s and (b) (b') p polarizations. The dashed line in panel shows a 2LO-replica spectrum of the occupancy function for polariton states of branch 1; this spectrum was used to calculate the distribution function $\mathrm{f} 1$ $(\omega)$, which is shown by a dotted line. (0) Total intensity and (triangles) experimental data, $(1-3)$ the contributions of the transverse polaritons (branches 1,2) and longitudinal excitons (branch 3), and (23) the interference contribution Ip23. 
important role in the formation of total intensity $I_{S}^{(0)}$ in the frequency range $\omega \geq \omega_{L}$.

We should especially note the following. As can be seen in Figure 4(a') and Figure $4\left(c^{\prime}\right)$, using renormalization of dashed curve 1 (describing the contribution $I_{1 S}^{(0)}$ of lower branch polaritons), one can obtain agreement between this curve and experiment with a certain error; however, within this error, it is not correct to put a question about the exact value of contribution $I_{2 S}^{(0)}$. Therefore, the good description of experimental results based on the kinetic theory in the studies devoted to the PL in the vicinity of longitudinal exciton frequency seems doubtful.

Figure 4(c) shows an EPL profile for a CdS crystal in the p-polarized emission geometry at $\theta \approx 80^{\circ}$; here, along with the fundamental maximum $A_{T}$, one can see a small additional maximum $A_{L}^{\prime}$, located at the short-wavelength side of $\omega_{L}$ (at the frequency $\omega_{\theta=80^{\circ}}=2.5547 \mathrm{eV} / \mathrm{h}$ ). Previously the occurrence of this maximum was explained by only the "deexcitation" of purely longitudinal excitons. However, according to the results of numerical calculations presented in Figure $4\left(\mathrm{c}^{\prime}\right)$, the $A_{L}^{\prime}$ line is formed in a complicated way. The spectral dependences of the total intensity $I_{p}^{(0)}$ (solid line 0 ) and the partial contributions $I_{1 p}^{(0)} \quad$ (curve 1), $I_{2 p}^{(0)} \quad(2), I_{3 p}^{(0)} \quad$ (3), and the interference component $I_{p 23}^{(0)} \quad$ (23) were calculated for the known CdS parameters [2] at the same $\Gamma, L$, and $\ell$ values as the curves in Figure 4(a') and Figure 4( $\left.\mathrm{c}^{\prime}\right)$. The frequency range $\omega<\omega_{\theta}$ is noteworthy; here, within the kinetic approximation, longitudinal excitons (as well as transverse polaritons 2) cannot be transformed into external photons, i.e., yield luminescence into vacuum. According to the wave theory, which is consistent with the experiment (compare curve 0 and triangles in Figure $4\left(c^{\prime}\right)$, the exciton decay induces emission of radiative surface modes and their interference. This, undoubtedly, is a new mechanism of EPL formation in the crystals having spatial dispersion. Strictly speaking, the frequencies $\omega_{\theta}$ are somewhat different for waves 2 and 3. At $\Gamma=0$ in the vicinity of $\omega_{L}$, the dispersion relations for waves 2 and 3 can be expressed accurate to the small parameter $\omega_{M} \varepsilon_{b} / \omega_{L T} \ll 1$ using the formulas

$$
\begin{gathered}
\omega_{2 \mathbf{k}}^{(T)} \approx \omega_{L}+n_{2}^{2}(\omega) \cdot \omega_{L T} / \varepsilon_{b}, \\
\omega_{\mathbf{k}}^{(3)} \approx \omega_{L}+n_{3}^{2}(\omega) \cdot \omega_{M} .
\end{gathered}
$$

Hence, (18) and (19) yield the following relations for the characteristic frequencies $\omega_{\theta}^{(2)}$ and $\omega_{\theta}^{(3)}$ :

$$
\begin{gathered}
\omega_{\theta}^{(2)} \approx \omega_{L}+\sin ^{2} \theta \cdot \omega_{L T} / \varepsilon_{b}, \\
\omega_{\theta}^{(3)} \approx \omega_{L}+\sin \theta \cdot \omega_{M} .
\end{gathered}
$$

In the case of ultimately large exit angles $\left(\theta \approx 85^{\circ}\right)$, these relations yield the following expressions for the CdS parameters at $T=2 \mathrm{~K}$ :

$$
\omega_{\theta, \max }^{(2)} \approx \omega_{L}+\omega_{L T} / \varepsilon_{b}, \quad \omega_{L T} / \varepsilon_{b} \approx 0.22 \mathrm{мэB},
$$




$$
\omega_{\theta, \max }^{(3)} \approx \omega_{L}+\omega_{M}, \hbar \omega_{M} \cong 7 \times 10^{-3} \text { мэВ, } \hbar \omega_{L}=2.5544 \text { эВ . }
$$

As was shown by the experiment and numerical calculation (Figure 4(c')), the $A_{L}^{\prime}$ emission line is peaking specifically near the characteristic frequency of transverse polariton $2: \hbar \omega_{\theta, \max }^{(2)} \approx 2.5546 \mathrm{eV}$. Due to the influence of exciton decay $\Gamma$, the real maximum of the frequency dependence $I_{3 p}^{(0)}$ shifts to shorter wavelengths with respect to the frequency $\omega_{L}$ and approaches the frequency $\omega_{\theta} \approx \omega_{\theta}^{(2)}$. Figure $4\left(c^{\prime}\right)$ shows also that the profile of the $A_{L}^{\prime}$ line cannot be obtained using only the contribution $I_{3 p}^{(0)}$. It should be especially noted that, even for the practically minimal value $\hbar \Gamma=0.075 \mathrm{meV}$ for CdS crystals, the kinetic approximation cannot be used to describe the partial contributions $I_{2 p}^{(0)}$ and $I_{3 p}^{(0)}$ in the vicinity of the emission line $A_{L}^{\prime}$, because $\left(\omega_{\theta \text {, max }}-\omega_{L}\right) / \Gamma \approx 2.0$ and inequality (1) is not satisfied at $\omega=\omega_{\theta}$ for the longitudinal wave, whereas for the transverse mode $\omega_{\theta}$ is a critical frequency, below which the $\boldsymbol{k}_{2}$ value is purely imaginary at $\Gamma=0$ for the given direction $\Omega(\theta, \varphi)$ in vacuum.

Figure 5 shows a comparison of the theoretical (curves 0 ) and experimental

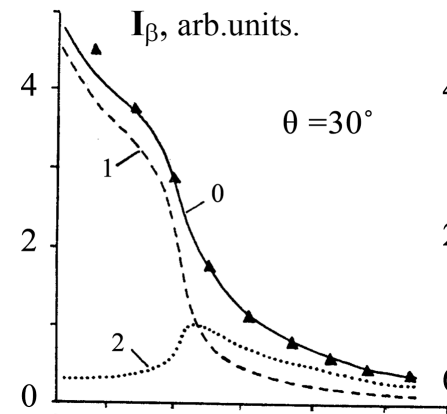

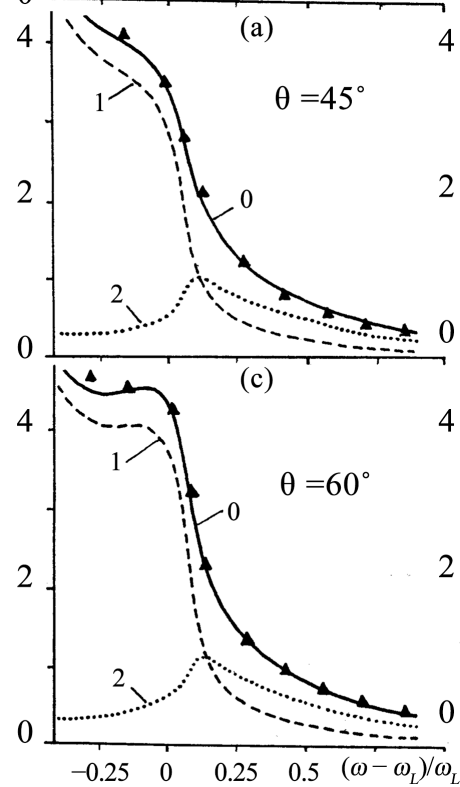

(e)

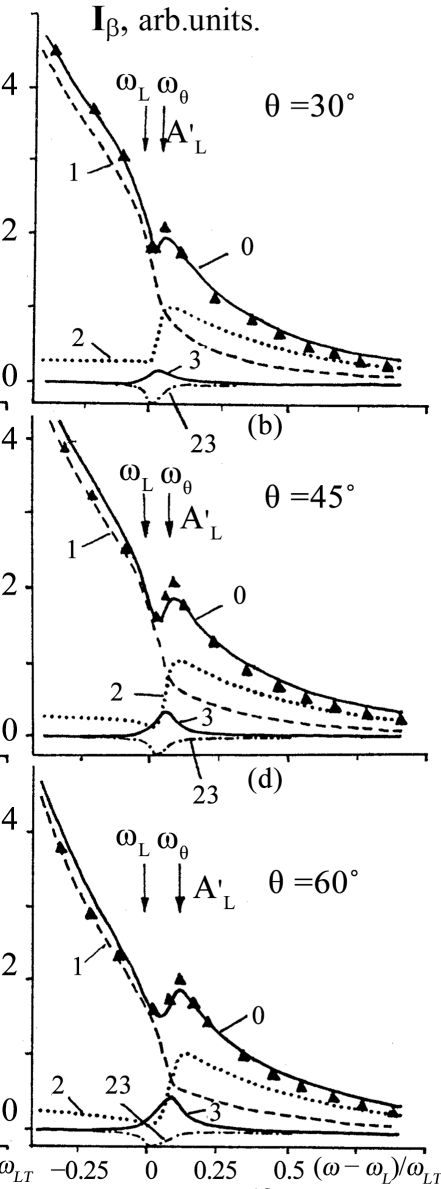

(f)

Figure 5. Comparison of the theoretical (curves 0) and experimental (triangles) EPL spectra for the (a) (c) (e) s- and (b) (d) (f) p-polarized emission from CdS crystals at different exit angles: $\theta=30^{\circ}, 45^{\circ}$, and $60^{\circ}$. The designations are the same as in Figure 4. 
(triangles) spectra of CdS crystals and the calculated frequency dependences of the partial contributions (curves $1,2,3,23$ ) in the emission geometries corresponding to the $\mathbf{s}(a, c, e)$ and $\mathrm{p}(b, d, f)$ polarizations at different values of the exit angle $\theta: 30^{\circ}(a, b), 45^{\circ}(c, d)$, and $60^{\circ}(e, f)$. One can see that the relative contributions $I_{1 \lambda}^{(0)}$ and $I_{2 \lambda}^{(0)}$ to the luminescence depend weakly on $\theta$, however, the contribution $I_{3 p}^{(0)}$ (curves 3 ) from normal wave 3 significantly increases with a rise in $\theta$ and plays a key role in the formation of the $A_{L}^{\prime}$ line.

The rise in the contribution $I_{3 p}^{(0)}$ with an increase in $\theta$ is directly related to the manifestation of SD in luminescence spectra. In the absence of SD, waves 3, as well as waves 1 , cannot exist in the frequency range $\omega>\omega_{L}$. At $\theta=0$, longitudinal waves 3 undergo total internal reflection and, therefore, the contribution of $I_{3 p}^{(0)}$ to $I_{p}^{(0)}$ is zero. This situation occurs also at $n_{3 L}>\sin \theta$, a fact explaining the sharp decrease in $I_{3 p}^{(0)}$ with an increase in frequency in the range $\omega>\omega_{\theta}$. It is noteworthy that the interference component $I_{p 23}^{(0)}$ gives a small negative contribution to the total intensity $I_{p}^{(0)}$, and its absolute value decreases with an increase in $\theta$, when modes 2-2' and 3-3' diverge in Figure 1. However, without the contribution of $I_{p 23}^{(0)}$, it is difficult to obtain quantitatively a small minimum at $\omega_{L}$ in the EPL spectrum that would be practically independent of $\theta$. Let us analyze in more detail the blue shift of the $A_{L}^{\prime}$ line with respect to $\omega_{L}$ that occurs with an increase in angle $\theta$ at $\omega_{M} \varepsilon_{b} \ll \omega_{L T}$ and $\Gamma \ll\left(\omega_{\theta}-\omega_{L}\right)$. In this case, taking into account that $n_{\beta}^{2}=n_{\beta z}^{2}+\sin ^{2} \theta$, the dispersion relation for the states of branch 2 (near $\omega_{L}$ ), emitting into vacuum, can be written as

$$
n_{z}^{2} \cong \varepsilon_{b}-\frac{\varepsilon_{b} \omega_{L T}^{\prime}}{\omega-\omega_{0}},
$$

where

$$
\omega_{L T}^{\prime}=\omega_{L T}+\frac{\omega-\omega_{0}}{\varepsilon_{b}} \sin ^{2} \theta \approx \omega_{L T}\left(1+\frac{\sin ^{2} \theta}{\varepsilon_{b}}\right) .
$$

Naturally, for the $\theta$ values at which $n_{z}^{2}<0$, wave 2 undergoes spatial decay even at $\Gamma=0$. As follows from (22), this occurs in the frequency range $\omega<\omega_{L}$ at $\theta=0$ and, at $\theta \neq 0$, in the frequency range below $\omega_{\theta}$, the formula for which can be derived from (22):

$$
\omega_{\theta}=\omega_{L}+\left(\frac{\sin ^{2} \theta}{\varepsilon_{b}-\sin ^{2} \theta} \omega_{L T}\right) .
$$

This expression differs from (20) by only the replacement of $\varepsilon_{b}$ with $\varepsilon_{b}-\sin ^{2} \theta$. Thus, with an increase in $\theta$, the bottom of the energy band of "light" transverse polaritons emitting into vacuum shifts upwards; i.e., the longitudinal transverse splitting $\omega_{L T}$ somewhat increases (see (23)). This effect manifests itself mainly in the shift of the $A_{L}^{\prime}$ emission line with an increase in $\theta$. For two exit angles, $\theta_{1}$ and $\theta_{2}$, formula (24) yields the relation

$$
\frac{\omega_{\theta 1}-\omega_{L}}{\omega_{\theta 2}-\omega_{L}}=\frac{\left(\varepsilon_{b} / \sin ^{2} \theta_{2}\right)-1}{\left(\varepsilon_{b} / \sin ^{2} \theta_{1}\right)-1} \text {. }
$$


Using this relation, we obtain a value of 3.2 for $\operatorname{CdS}\left(\varepsilon_{b}=9.4\right)$ at $\theta_{1}=60^{\circ}$, $\theta_{2}=30^{\circ}$, whereas Figure 5(b), $(f)$ demonstrates a somewhat different value: 2.8 . The cause of this small inconsistency is that the condition $\Gamma \ll\left(\omega_{\theta}-\omega_{L}\right)$ is not exactly satisfied in the case under consideration.

\section{Conclusions}

1) A theory of exciton-polariton luminescence of cubic crystals with spatial dispersion, when the applicability criteria of the kinetic Boltzmann equation for the geometry of longitudinal-exciton emission are violated, was developed. A new mechanism of EPL formation was proposed, which involves coherent scattering of polaritons from well-defined initial quantum states into emitting (final) states of normal modes with a short lifetime, for which the wave vector is not a well-defined quantum number.

2) The formation of EPL spectrum has a number of new specific features. Due to the transformation of the normal-wave spectrum with allowance for the real exciton decay, radiative surface modes are involved in the energy transfer through the crystal boundary, and the presence of SD leads to their interference interaction. The contributions of $I_{3 p}^{(0)}$ and $I_{23 p}^{(0)}$ to $I_{p}^{(0)}$ sharply decrease with an increase in $\hbar \Gamma$, which can be explained by the SD suppression due to mechanical-exciton decay for longitudinal waves. At the same time, the contribution of $I_{2 p}^{(0)}$ increases and becomes significantly broadened, as a result which an increase in $\Gamma$ at the same $L$ value leads to a weak decrease in the amplitude of the $I_{p}^{(0)}(\omega)$ peak at $\omega_{\theta}$ and its broadening.

3) The contribution of purely longitudinal excitons to the EPL and their interference with polaritons of the upper dispersion branch are low ( 10-20\%); nevertheless, they must be taken into account to obtain quantitative agreement with experiment.

4) The above-described method for analyzing EPL spectra makes it possible to determine the optical parameters of crystal (for example, the $\omega_{L T}$ value proceeding from the frequency of the $A_{L}^{\prime}$ line). It can be used with small variations to study the inelastic scattering spectra of polaritons in the vicinity of longitudinal exciton frequency (taking into account the finite decay and $\mathrm{SD})$.

\section{Acknowledgements}

We are grateful to A.V. Sel'kin for supplying experimental results.

\section{References}

[1] Benoit a la Guillame, C., Bonnot, A. and Delever J.M. (1970) Luminescence from Polaritons. Physical Review Letters, 24, 1235-1238. https://doi.org/10.1103/PhysRevLett.24.1235

[2] Cross, E.F., Permogorov, S.A., Travnikov, V.V. and Sel'kin, A.V. (1972) Polariton Emission from Crystals. Solid State Communications, 10, 1071-1074. https://doi.org/10.1016/0038-1098(72)90898-8 
[3] Permogorov, S.A., Travnikov, V.V. and Sel'kin, A.V. (1972) Effects of a Spatial Dispersion in Reflexion Spectra at Inclined Falling of Light on Crystal Border. Physics of the Solid State, 14, 3642-3651.

[4] Sel'kin, A.V. (1977) Energy Transfer by the Normal Modes in the Exciton Resonance Region. Physica Status Solidi (b), 83. 47-53.

https://doi.org/10.1002/pssb.2220830103

[5] Ivchenko, E.L., Pikus, G.E. and Yuldashev, N.Kh. (1981) Transfer of the Polarized Radiation in Crystals in Exciton Spectrum Areas. Polariton Effects. JETP, 80, 1228-1246.

[6] Abdukadirov, A.G., Ivchenko, E.L., Sajin, M.I., Sel'kin, A.V. and Yuldashev, N.Kh. (1989) Поляритонная люминесценция в окрестности частоты продольного экситона с учетом затухания. Optics and Spectroscopy, 67, 845-850.

[7] Abdukadirov, A.G., Sajin, M.I., Sel'kin, A.V. and Yuldashev N.Kh. (1990) Polariton Luminescence of the Mixced Modes in Crystals with a Spatial Dispersion. JETP, 97, 644-662.

[8] Yuldashev, N.Kh. (2001) Exciton-Polariton Luminescence and Transfer of Resonant Radiation in Crystals. Ferghana, 214.

[9] Akhmadaliev, B.J., Polvonov, B.Z. and Yuldashev, N.Kh. (2014) Influence of Exciton Decay on the Polariton Luminescence Spectra of CdTe Crystal. Optics and Spectroscopy, 116, 244-248. http://www.ioffe.ru/journals/os/

[10] Permogorov, S.A., Surkova, T.P. and Tenishev, A.N. (1998) Exciton Luminescence of $\mathrm{Cd}_{1-\mathrm{x}} \mathrm{Fe}_{\mathrm{x}} \mathrm{Te}$ Solid Solution. Physics of the Solid State, 40, 897-901. http://journals.ioffe.ru/articles/33939

[11] Bagaev, V.S., Klevlov, Yu.V., Kolosov, S.A., Krivobok, V.S. and Shepeli, A.A. (2010) Optical and Electrophysical Properties of Defects in High-Purity CdTe. Physics of the Solid State, 52, 37-42. http://journals.ioffe.ru/articles/1677 https://doi.org/10.1134/S1063783410010075 